\title{
Desafíos territoriales de la construcción de paz en Colombia
}

\author{
Territorial challenges of peacebuilding in Colombia \\ - Ava Gómez Daza y Adriana Romero Sánchez \\ Universidad de Salamanca y University of Wisconsin-Madison
}

DOI: http://dx.doi.org/10.15304/ricd.3.14.7745

\section{NOTAS BIOGRÁFICAS}

Ava Gómez Daza es doctora en Ciencia Política por la Universidad de Salamanca (España), magister en Estudios Latinoamericanos y licenciada en Comunicación Audiovisual y en Sociología por la misma universidad. Realizó posdoctorado en Ciencias Sociales en la Universidad de Buenos Aires (UBA) con beca Conicet-UBA.

Contacto: avagomez@usal.es

Adriana Romero Sánchez es estudiante del programa LLM/SJD de la Universidad de WisconsinMadison (Estados Unidos) y becaria Minciencias-Fulbright. Magíster en Sociología y Política Criminal, analista espacial y abogada de la Universidad Nacional de Colombia.

Contacto: romerosanchez@wisc.edu

\section{Resumen}

El presente ensayo recoge algunas reflexiones sobre los retos territoriales de la implementación de Acuerdo Final para la Terminación del Conflicto y la Construcción de una Paz Estable y Duradera entre el Gobierno colombiano y las FARC. El texto profundiza en las dimensiones del acuerdo que han tenido mayores dificultades para su implementación y en las implicaciones territoriales que se derivan de los retrocesos resultantes. Concluimos reflexionando sobre el Paro Nacional de 2021 donde resurgen las demandas de una sociedad más justa, igualitaria y democrática. La respuesta institucional, orientada principalmente a reprimir a las ciudadanas, pone de manifiesto que las instituciones del Estado colombiano siguen estando muy lejos del camino de diálogo y reconciliación labrado después de la firma de los acuerdos de paz en 2016.

\section{Abstract}

This essay explores some territorial challenges related to the implementation of the Final Agreement for the Termination of the Conflict and the Construction of a Stable and Lasting Peace between the Colombian Government and the Revolutionary Armed Forces of Colombia (FARC). In this text, we aim to delve deeply into the critical points of the agreement, which have encountered insurmountable setbacks that have had a considerable impact on the territories. We conclude by reflecting on the National Strike of 2021 where resurfaced the demands of a more just, equal, and democratic society. The institutional response, mainly aimed at suppressing citizens, shows that Colombian institutions are still far from the path of dialogue and reconciliation shaped by the Peace Agreement of 2016.

\section{Palabras clave}

Colombia, Acuerdo de Paz, movilización social, juventud. 
Keywords

Colombia, Peace Agreement, social mobilization, youth.

\section{Sumario}

1. Introducción

2. El fantasma de la aspersión aérea y el retroceso en la política de la solución al problema de las drogas ilícitas

3. Masacres y asesinatos selectivos (¡nos están matando!)

4. Denuncia y resistencia: los medios como alternativa de la sociedad civil frente a la reconfiguración de violencia

5. El paro nacional de 2021 y la eclosión de todas las luchas

\section{Contents}

1. Introduction

2. The specter of aerial spraying and the setback in the policy of solving the problem of illicit drugs

3. Massacres and targeted killings (they are killing us!)

4. Denunciation and resistance: the media as an alternative for civil society in the face of the reconfiguration of violence

5. The national strike of 2021 and the emergence of all the struggles 


\section{INTRODUCCIÓN}

A casi cinco años de la firma de los acuerdos de paz entre el gobierno colombiano y las FARC$E P$, la reflexión en torno a su implementación surge al calor de un clima de alta polarización, desinformación, y movilización social agravado por el contexto de pandemia mundial. Los atentados en contra de la vida de líderes y lideresas sociales, y en contra de las personas firmantes de los acuerdos, así como las decisiones y políticas regresivas en asuntos como la solución al problema de las drogas de uso ilícito, evidencian un panorama complejo que se puede agravar si desde la política central se continúa dando la espalda al nivel territorial y afrontando dichas conflictividades con la tradicional e inefectiva militarización de la sociedad.

La victimización de liderazgos sociales, que refleja un claro retroceso en la implementación de los acuerdos de paz, no es ni de lejos el único de los obstáculos que han surgido en el marco de la implementación de los acuerdos. Tampoco los debates alrededor del problema de la cocaína y el aumento de las hectáreas de cultivos de coca. A pesar de los avances al garantizar el desarme y el proceso DDR de los excombatientes, así como la implementación del Sistema Integral de Verdad, Justicia, Reparación y Garantías de No Repetición, son múltiples los nuevos desafíos que la implementación del Acuerdo Final para la Terminación del Conflicto y la Construcción de una Paz Estable y Duradera, entre los que pueden destacarse los relacionados con la reforma rural integral, el desmonte del paramilitarismo o la participación política1.

Estos aspectos evidencian de manera clara los padecimientos que los territorios viven cinco años después con la implementación de los acuerdos, que si bien son problemas que ameritan un análisis profundo y que revisten de características y dimensiones particulares, en esta reflexión queremos proponer dos puntos de articulación, que son buen ejemplo para pensar en la posibilidad de cumplir el objetivo 16 de desarrollo sostenible (paz, justicia e instituciones sólidas). El primero, la abismal distancia entre el gobierno central y tomador de decisiones respecto del camino que se debe recorrer para resolver problemas complejos en zonas donde el Estado ha fallado en hacer presencia; el segundo, la importancia para la sociedad civil de disponer de herramientas de comunicación alternativas para denunciar violaciones de derechos humanos y afectaciones al territorio, que se producen y acrecientan un clima de conflictovidad social.

\section{EL FANTASMA DE LA ASPERSIÓN AÉREA Y EL RETROCESO EN LA POLÍTICA DE LA SOLUCIÓN AL PROBLEMA DE LAS DROGAS ILÍCITAS}

La transición hacia la aspersión aérea como solución al problema de las drogas ilícitas vuelve al ruedo en la política nacional con el anuncio del Decreto 380 de 20212, que fija los criterios para el retorno de la aspersión aérea con glifosato, después de seis años de suspensión.

La avanzada en la política de aspersión aérea como forma de acabar con los cultivos de uso ilícito es un retroceso en materia del reconocimiento de la problemática estructural asociada a los cultivos. La decisión del gobierno marca una regresión hacia una política antidroga desconocedora de la dimensión social de la problemática que se intentó paliar con el Programa Nacional Integral de Sustitución de Cultivos de Uso llícito (PNIS) y la Reforma Rural Integral (que se articularon en los puntos 1 y 4 de los acuerdos de paz).

En este sentido, las alternativas propuestas por el acuerdo de paz se centraron en el planteamiento de una política con potencial de tener impactos sostenibles en el mediano y largo plazo, otorgando opciones y garantías a las familias para transitar hacia una economía lícita. Los problemas de la decisión regresiva en materia de lucha contra las drogas no solo se relacionan con los impactos en la salud de las comunidades en zonas asperjadas por un químico que es potencialmente cancerígeno y destructor de la fauna y flora donde recae ${ }^{3}$, sino que, además, el grave impacto social que produce no necesariamente acaba con el negocio del narcotráfico:

El negocio de los narcotraficantes se afecta solo marginalmente, pero se generan grandes impactos sociales como el desplazamiento de comunidades vulnerables que se ven obligadas a reasentarse en nuevas zonas para resembrar ante la necesidad de obtener ingresos para sus familias. Esto intensifica aún más el conflicto social y las tensiones entre las comunidades y el Estado debilitando la imagen y legitimidad del mismo en los territorios a pesar de que la estrategia propuesta en los acuerdos de paz distaba claramente de esta alternativa como solución al problema de las drogas $^{4}$. 
Los escasos avances en materialización de los acuerdos de paz en los puntos 1 y 4 se aúnan a las enormes dificultades que persisten en cuanto a la consolidación de una infraestructura que permita al campesinado transformar su actividad:

Según la Encuesta de Diagnóstico del hogar para la renovación y transformación integral, realizada por UNODC, el $45 \%$ de las 3.543 familias que hacen parte del PNIS [Programa Nacional Integral de Sustitución de Cultivos de Uso Ilícito], afirman no contar con vías de acceso terrestre. Quienes afirman contar con vías, el $48 \%$ dice que el estado de las vías es "regular" y el $36 \%$ "malo" 5 .

En contravía de la posición vanguardista que Colombia había adoptado en el escenario internacional, como uno de los principales afectados por las políticas de lucha contra las drogas ${ }^{6}$, y que estaba logrando un eco en transformar las agendas en esta materia7, actualmente se están revisitando medidas que han evidenciado tener efectos situacionales sobre el número de hectáreas cultivadas, a un costo humano, institucional, económico irrazonable, y que no atiende las raíces del problema: la presencia de actores que ejercen el control territorial para fines políticos o criminales ${ }^{8}$.

\section{MASACRES Y ASESINATOS SELECTIVOS (¡NOS ESTÁN MATANDO!)}

El ejercicio de la violencia en contra de los firmantes de paz, los líderes y lideresas, y personas en el ejercicio de la política desde la oposición, muestran los importantes desafíos y la escasa voluntad del actual gobierno de hacer frente a estos problemas. Uno de los síntomas de la imposibilidad de llevar a cabo acciones contundentes, que respondan a las demandas de protección y de defensa de la vida, está asociado con el uso de eufemismos, la retórica sobre los atributos de las víctimas o discusiones alrededor de los móviles que orientan estos homicidios.

En los últimos meses, el presidente Iván Duque insistió que lo que se estaba viviendo en los territorios no eran masacres, sino homicidios colectivos $^{9}$. El argumento principal de no utilizar una categoría ampliamente reconocida en la literatura de los derechos humanos y la criminología, e incluso presente en la legislación, era que el término no se encontraba consagrado en el Código penal. Como tampoco lo están los homicidios selectivos. Sin embargo, este eufemismo generó una amplia polémica en medios, en un momento en que, con escasos días de distancia, se estaban presentando de manera preocupante asesinatos de jóvenes, principalmente en el Pacífico colombiano. Dicha discusión se dio de la mano de la presentación de estadísticas, en las que señaló que los hechos de "homicidios colectivos" apenas representaban un $2 \%$ del total de casos en los últimos 22 , un intervalo temporal bastante atípico para calcular datos ${ }^{10}$. Además de normalizar el fenómeno y señalar que esto ya ocurría el pasado, dicha estrategia no sólo buscaba relativizar la responsabilidad del gobierno frente a los hechos, sino despojar los hechos de sus connotaciones políticas y las graves indicaciones de estar viviendo una situación grave en materia de derechos humanos ${ }^{11}$.

Por su parte, en la última década se han resaltado las apremiantes situaciones de violencia que han padecido los defensores de derechos humanos y líderes sociales ${ }^{12}$. En particular, de 2016 a 2020, de acuerdo con cifras de la Oficina del Alto Comisionado de las Naciones Unidas para los Derechos Humanos, más de 400 defensores de derechos humanos han sido asesinados $^{13}$. Sin embargo, a pesar de las masivas denuncias, el Gobierno Nacional, principalmente en cabeza del Ministerio del Interior ha señalado que durante el gobierno de Iván Duque se han venido reduciendo estos crímenes contra esta población ${ }^{14}$, y que los últimos años, al contrario de empeorar la situación, el gobierno ha contribuido a la protección y el Estado ha cumplido su obligación de investigar, judicializar y condenar a los responsables. Sin embargo, incluso las cifras institucionales difieren de esta supuesta mejoría, como lo resalta el informe sobre homicidios contra líderes sociales y defensores de derechos humanos ${ }^{15}$. Dentro de las principales justificaciones que se encuentran cuando los registros institucionales no coinciden están, por ejemplo, que la persona no se encontraba activa ejerciendo la labor de la defensa de los derechos humanos ${ }^{16}$. También, se omiten otras fuentes de contraste ${ }^{17}$. $Y$, finalmente, se alega en el discurso oficial que los asesinatos en particular están asociados con móviles personales, como "los líos de faldas"18.

Esta realidad genera rabia, impotencia y zozobra en la sociedad civil, pero en particular a quienes habitan los territorios en donde todavía se disputa el control de actores armados e intereses plurales. Sin embargo, los deficientes modelos de protección, de la mano de discusiones teóricas áridas y un escaso control de la violencia, reproducen También, la frustración de un acuerdo incumplido, capaz de reflejar las 
problemáticas estructurales de violencia y los caminos que debería recorrer el país para su superación, deja una huella imborrable de desconfianza institucional, de desilusión y desesperanza sobre la posibilidad de construir una sociedad pacífica y prometedora.

\section{DENUNCIA Y RESISTENCIA: LOS MEDIOS COMO ALTERNATIVA DE LA SOCIEDAD CIVIL FRENTE A LA RECONFIGURACIÓN DE VIOLENCIA}

El primer lustro de la firma de los acuerdos ha demostrado, no obstante, el papel central de la sociedad civil en la denuncia de las masacres cometidas en contra de la ciudadanía. No es una novedad que los medios de comunicación de la sociedad civil hayan sido los encargados de exponer públicamente las dimensiones de la violencia a lo largo del conflicto armado:

En el contexto del conflicto colombiano, a partir de mediados de los años $70-y$ con especial énfasis durante la década de los 90- surgen innumerables experiencias ciudadanas, alternativas, comunitarias, de personas y colectivos organizados en la construcción de interacciones mediadoras con su propia necesidad. Venciendo las limitaciones establecidas durante los periodos más cruentos de la represión y despolitización de la sociedad civil, los medios comunitarios consiguieron convertirse en muchas ocasiones en creadores de sentido social. Es decir, si bien, en un primer momento, tal y como recogen los estudios de antropología de la violencia, el conflicto armado "hace estallar las redes de significado que tanto individuos como colectividades utilizan para darle sentido a la vida cotidiana"19, en momentos posteriores de ruptura e indignación, en la crisis de legitimidad presente en los diferentes bloques históricos, desde la sociedad civil organizada la comunicación y la cultura pueden ser entendidas también como herramientas de resistencia y lucha contra el conflicto armado 20 .

Después de la firma de los acuerdos de paz, han sido precisamente los medios de comunicación de la sociedad civil los que han puesto de manifiesto las formas de violencia desatadas, en particular, en contra de los líderes y lideresas sociales a nivel territorial, en una ola de violencia con pocos precedentes en el país. En el marco de los debates como el presentado en la sección anterior, la existencia de mecanismos de información continua y confiable sobre la violencia resulta una necesidad. Pacifista, por ejemplo, es un medio que ha sido revelador de la situación que se vive a nivel territorial, contando - desde el inicio de la implementación de los acuerdos- los líderes y lideresas que han sido asesinados. Un conteo del que se desprende la escalofriante cifra de $387^{21}$ muertes que, lejos de ser solo un número, refleja la desarticulación de procesos sociales organizativos orientados a la defensa de la paz a nivel territorial, expresa la victimización de cientos de familiares, niños y niñas que se suman a las víctimas de la violencia en un país que hizo la paz.

El trabajo de las emisoras de la sociedad civil ha sido perenne en la denuncia de violaciones a los derechos humanos, incluso por parte de los agentes del Estado, en cuyo caso, el trabajo de Violeta Estéreo ha expuesto de forma extensiva a lo largo de los últimos años los hechos de ejecuciones de civiles desarmados por agentes del Estado que ilegítimamente los presentaron como guerrilleros dados de baja en combate. Al respecto, es ilustrativa la entrevista realizada por esta emisora al expresidente Álvaro Uribe Vélez, dos años después de que se conocieran los primeros casos de lo que hoy organizaciones no gubernamentales como Human Rights Watch ${ }^{22}$ reconocen como una práctica sistemática del Ejército colombiano durante su gobierno:

Eso es falso, eso es falso. Eso lo han dichos los enemigos de la Seguridad Democrática. La Contraloría General de la Nación revisó y no hay un solo peso de recompensas pagado, que tenga que ver con falsos positivos. Díganlo nacionalmente. Eso lo han dichos algunas personas enemigas de la Seguridad Democrática23.

A pesar de ello, los esfuerzos resilientes de organizaciones civiles por dar a conocer las diversas dimensiones de violencia que persiste tras varios años del inicio de la implementación de los acuerdos de paz han permitido que organizaciones internacionales pongan la lupa sobre los limitados avances en la protección de los líderes sociales y las personas firmantes de los acuerdos en Colombia. Al respecto, el relator especial de derechos humanos se pronunció sobre la grave situación que viven los líderes y lideresas sociales en Colombia de la siguiente forma:

20. Desde 2016, hasta el 30 de junio de 2019, Colombia sigue siendo el país con el mayor índice de asesinatos de personas defensoras de derechos humanos en América Latina, en base a los casos recopilados y verificados por las Naciones Unidas, y con un alto índice de amenazas, ataques, desplazamientos y otras violaciones de los derechos de personas defensoras. Las personas defensoras son asesinadas y violentadas por 
implementar la paz, oponerse a los intereses del crimen organizado, las economías ilegales, la corrupción, la tenencia ilícita de la tierra y por proteger sus comunidades. Las defensoras son además objeto de violaciones específicas de género y sus familias son también el blanco de ataques.

21. Si bien la tasa general de homicidios en Colombia ha seguido disminuyendo desde 2011 y desde la firma del Acuerdo de Paz (salvo un ligero ascenso en 2018), sigue siendo alta. Dicho descenso contrasta con el aumento de los asesinatos de personas defensoras, incluyendo los líderes y lideresas sociales, desde 2016, que fuentes estatales (Defensoría del Pueblo), de las Naciones Unidas (ACNUDH) y de la sociedad civil (por ejemplo, el Programa Somos Defensores) reportan (...) ${ }^{24}$.

De su parte, el contrapeso a la labor de las iniciativas de la sociedad civil para poner de manifiesto las violaciones de derechos humanos ha conllevado el esfuerzo determinante de algunas corporaciones mediáticas del país para el establecimiento de discursos negacionistas de las actividades criminales de determinados actores armados en el conflicto ${ }^{25}$, que han dado pie a que se instale en la opinión pública un álgido cuestionamiento de las instituciones encargadas de llevar a cabo la titánica labor de llevar la verdad de lo acaecido en el conflicto tanto a las víctimas como a la sociedad.

\section{EL PARO NACIONAL DE 2021 Y LA ECLOSIÓN DE TODAS LAS LUCHAS}

El Paro Nacional que inició en abril de 2021 reflejó lo convulsa que permanece la sociedad colombiana a varios años de la firma de los acuerdos de paz. La nonata reforma tributaria con la que el Ejecutivo pretendía capear la crisis devenida de la pandemia y de las decisiones fiscales pasadas orientadas a desgravar al sector empresarial ${ }^{26}$, fue la chispa que encendió a la ciudadanía que, desde el 28 de abril de 2021, inició un proceso de movilización masiva, con escasos precedentes en la historia colombiana.

Las movilizaciones, fundamentalmente aupadas por jóvenes y universitarios, recogieron no solamente las demandas orientadas a evitar reformas fiscales que ahondaran en las enormes deficiencias económicas de los sectores sociales medios y medios bajos, sino que abrieron toda una caja de pandora de demandas insatisfechas, hartazgo colectivo, incertidumbre, indignación, desigualdad, pobreza y rabia, contra una institucionalidad y unos representantes que se encuentran muy lejos de la realidad de la ciudadanía 27 .

La ciudadanía ha sido reprimida de forma violenta por la Fuerza Pública (Ejército y Policía) que ha saldado a la fecha, de acuerdo a la información recabada por la ONG Temblores, con al menos, 2.387 casos de violencia policial, entre los cuales registran 33 víctimas de agresión en sus ojos y 146 casos de disparos con armas de fuego por parte de la policía a civiles desarmados ${ }^{28}$. Asimismo, la Defensoría del Pueblo ha trasladado a la Fiscalía 43 reportes de homicidios en medio de las movilizaciones ${ }^{29}$. Al respecto se pronunció la CIDH señalando que el uso de la fuerza en contra de los manifestantes ha sido absolutamente desproporcionado ${ }^{30}$.

Los jóvenes -quienes encabezan las movilizaciones- han hecho propias las demandas de una sociedad más justa, igualitaria y democrática, coincidentes con los propósitos centrales de los acuerdos de paz. Sin embargo, la represión y la violencia administradas con vigor en su contra recuerdan que la celebración del primer lustro de los acuerdos coincide con un periodo aciago de regresión hacia las retóricas represivas de principios del siglo XXI, en un país que arrastra enormes deudas sociales y retos de la implementación de paz a nivel territorial que aun lastran el avance de la sociedad hacia un camino de reconciliación.

\section{NOTAS}

1 En particular, desde una óptica política, los avances han sido limitados en materia de reforma rural integral, la deficitaria protección de personas en riesgo, las dificultades con los programas de sustitución, así como los retrasos en IOS PDET. Uno de los incumplimientos que ha ocupado un lugar central en la agenda pública es el rearme de las disidencias de las FARC, que trajo como consecuencia su expulsión del sistema de justicia transicional (así, la Sala de Reconocimiento, el 29 de agosto de 2019, expulsó a Luciano Marín Arango, alias Iván Márquez, José Manuel Sierra, alias Zarco Aldinever, y Henry Castellanos, alias Romaña; y la Sección de Apelación, el 13 de septiembre de 2019, expulsó a Seuxis Paucias Hernández Solarte, alias Jesús Santrich, y a Hernán Darío Velásquez Saldarriaga, alias El Paisa. En este sentido se pronunció la canciller Claudia Blum ante la ONU en abril de 2021 (Ver: Caracol Radio, "Canciller: 'debe considerarse disidencias como incumplimiento al acuerdo'”, 22 de abril de 2021. Disponible en

https://caracol.com.co/radio/2021/04/22/nacional/16190 65164 772793.html). A pesar de los graves efectos que esto tiene sobre la construcción de confianza, reconciliación y paz, no puede equipararse con las obligaciones institucionales en cabeza del Estado, se podría afirmar que los cumplimientos robustos del Acuerdo han estado orientados a garantizar el desarme y el proceso DDR de los 
excombatientes, así como la implementación del Sistema Integral de Verdad, Justicia, Reparación y Garantías de No Repetición. Sobre las alertas del proceso de implementación, véase el informe de la Procuraduría General de la Nación al Congreso de la República, presentado en septiembre de $2020 . \quad$ Disponible en https://www.procuraduria.gov.co/portal/media/file/Segundo \%20Informe\%20al\%20Congreso\%20Paz\%20-

\%20Procuradur\%C3\%ADa\%20General\%20de\%20la\%20Naci \%C3\%B3n.pdf

2 Decreto 380 del 12 de abril de 2021. Disponible en https://img.lalr.co/cms/2021/04/12221401/DECRETO380-DEL-12-DE-ABRIL-DE-2021.pdf.

3 Universidad de los Andes, "Aspersión aérea: problemas de salud e incremento de la violencia", 13 de abril de 2021. Disponible

https://uniandes.edu.co/es/noticias/economia-y-

negocios/aspersion-aerea-problemas-de-salud-eincrementode-violencia.

${ }^{4}$ Rubiano, M.J. et al. (2020) “¿Reanudar la fumigación aérea de cultivos ilícitos en Colombia? Un resumen de la literatura científica", Comentario de Política \# 2. CESED - Universidad de los Andes, pág. 14. Disponible en https://cesed.uniandes.edu.co/wp-

content/uploads/2021/03/Reanudar-fumigaci\%C3\%B3n-decultivos_.pdf.

5 Garzón J.C. y Gélvez, D. (2018) “¿En qué va la sustitución de cultivos ilícitos? La implementación, los rezagos y las tareas pendientes. Informe 04 - FIP. Disponible en http://ideaspaz.org/media/website/FIP_sustitucion_final.pdf

6 Por lo anterior, se abogaba por un giro de una política antinarcóticos a una política de salud pública. Un interesante análisis sobre su relación con el proceso de paz, sus impactos y perspectivas desde el lente estadounidense, como uno de los principales patrocinadores de la lucha antidrogas del país, puede consultarse en Beitel, J.S. y Rosen, L.W. Colombia's Changing Approach to Drug Policy, Congressional Research Services, noviembre de 2017. Disponible en https://fas.org/sgp/crs/row/R44779.pdf.

7 Entre otras rutas, siguiendo la trazada por la Comisión Asesora para la Política de Drogas en 2013 y 2015. Disponible

http://www.odc.gov.co/Portals/1/comision_asesora/product os.html.

8 Comisión Asesora para la Política de Drogas en Colombia, Lineamientos para un nuevo enfoque para la política de drogas en Colombia. Documentos técnicos, Bogotá, Ministerio de Justicia y del Derecho, mayo de 2015, págs. 115-119. Disponible en http://www.odc.gov.co/Portals/1/comision_asesora/docs/d ocumentos_tecnicos_comision_asesora.pdf.

Esta situación ya había sido advertida por muchos analistas, y existía una ruta para mitigar esa reconfiguración de los poderes armados ilegales en los territorios en donde la presencia del Estado no ha logrado tener la misma respuesta que en ocasiones han ocupado dichos actores. Entre otros, véase Valencia, L y Ávila, A. (2016) Los retos del postconflicto. Justicia, seguridad y mercados ilegales. Bogotá, Ediciones B.

9 Umaña, C., “Homicidios colectivos”: un estado de negación, Revista Razón Pública, 31 de agosto de 2020. Disponible en https://razonpublica.com/homicidios-colectivos-estadonegacion/. Aquí puede encontrarse un interesante análisis sobre el desafortunado eufemismo utilizado por el Gobierno Nacional.
10 Cifras disponibles en https://www.dw.com/es/duque-encolombia-no-hay-masacres-sino-homicidios-colectivos/a54662098.

11 En este sentido, González Posso en entrevista con Semana (Ver: Semana, "¿Cuál es la diferencia entre homicidios colectivos y masacres?", 24 de agosto de 2020. Disponible

https://www.semana.com/nacion/articulo/cual-es-ladiferencia-entre-homicidios-colectivos-y-masacres/696762/.

12 Comisión Interamericana de Derechos Humanos (2019). Personas defensoras de derechos humanos y líderes sociales en Colombia, pág. 15. Disponible en: http://www.oas.org/es/cidh/informes/pdfs/DefensoresColo mbia.pdf.

13 Un análisis más detallado puede encontrarse en Human Rights Watch (2021). Líderes desprotegidos y comunidades indefensas. Asesinatos de defensores de derechos humanos en zonas remotas de Colombia. Disponible en: https://www.hrw.org/sites/default/files/media_2021/02/co lombia0221sp_web.pdf.

14 En distintos momentos se ha mencionado por el Gobierno que se han reducido las cifras sobre este flagelo y se han resaltado los esfuerzos de esclarecimiento. Uno de esos momentos es discutido por Rozo, V y Uprimny, R. (2020). "Errores fatales: sobre la supuesta reducción de asesinatos de líderes con Duque". Disponible en: https://lasillavacia.com/opinion/errores-fatales-sobresupuesta-reduccion-asesinatos-lideres-duque-72926.

15 Consejería Presidencial para los derechos humanos y asuntos internacionales (2020). Informe de homicidios contra líderes sociales y defensores de derechos humanos 2016-2019. Disponible en: http://www.derechoshumanos.gov.co/Prensa/2020/Docum ents/Informe\%2OHomicidios\%20I\%C3\%ADderes\%20\%20Corte(14\%20Enero\%20de\%202020).pdf?TSPD_101_R0 $=0883 a 32 \mathrm{c} 4 \mathrm{dab} 2000 \mathrm{f} 172482 \mathrm{~b} 26 \mathrm{~b} 52 \mathrm{a0} 11 \mathrm{~b} 1 \mathrm{da00} \mathrm{d} 9 \mathrm{eOf} 4$ 335d0e6edfeca7fcfd42b47dced7c186b89089244048214 300002bcd4a73664ca56e5aaa8ca8564df87fc45b87103 25d925f214d1205f41145dbd0bc8723ab74f76ac5b9a47 41035a70. Sobre los cuestionamientos a estas cifras, véase Rozo, V y Uprimny, R., Ibíd.

16 Solo a modo de ejemplo, véase el comunicado del Ministerio del Interior de 18 de mayo de 2020, en el que señalan que una de los criterios de verificación de las cifras es que la labor de defensa de derechos humanos se encontrara activa al momento del homicidio. Comunicado disponible en: https://www.mininterior.gov.co/sala-deprensa/noticias/comunicado-de-prensa-4.

17 Rozo, V y Uprimny, R., Ibíd.

18 González, C. "Manipular las cifras sobre asesinato de líderes también es violencia”, Revista Razón Pública, 1 de marzo de 2021, Disponible en: https://razonpublica.com/manipular-las-cifras-asesinatolideres-tambien-violencia/

19 Rodríguez, C., “Introducción”, en Clemencia Rodríguez (Ed.), Lo que le vamos quitando a la guerra. Medios ciudadanos en contextos de conflicto armado en Colombia, Bogotá, Friedrich Ebert Stiftung, 2008, págs. 9-14.

20 Gómez , A. y Ramos, J. (2016) "Medios de comunicación en Colombia. Del conflicto al posconflicto", en Diego Bojarro Valiña et al. (Ed.), en América Latina y el Caribe, entre la encrucijada hemisférica y los nuevos retos globales, págs. 258-259.

21 Conteo realizado por Pacifista, intervalo: 1 de diciembre de 2016 a 27 de mayo de 2021. Disponible en

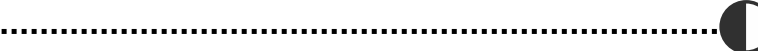


ENSAYOS

https://pacifista.tv/notas/lideres-sociales-asesinados-inicioimplementacion/.

22 HRW (2015) "El rol de los altos mandos en falsos positivos. Evidencias de responsabilidad de generales y coroneles del Ejército colombiano por ejecuciones de civiles". Disponible en https://www.hrw.org/es/report/2015/06/23/el-rol-de-losaltos-mandos-en-falsos-positivos/evidencias-deresponsabilidad-de.

23 Entrevista disponible en http://historico.presidencia.gov.co/sp/2010/marzo/24/022 42010.html.

24 Informe del Relator Especial sobre la situación de los defensores de los derechos humanos. Visita a Colombia. Consejo de Derechos Humanos [43er período de sesiones, 24 de febrero a 20 de marzo de 2020]. Tema 3 de la agenda 'Promoción y protección de todos los derechos humanos, civiles, políticos, económicos, sociales y culturales, incluido el derecho al desarrollo'. Disponible en https://undocs.org/es/A/HRC/43/51/Add.1.

${ }_{25}$ Revista Semana, "Carta a la JEP sobre algunas mentiras", 15 de noviembre de 2020. Disponible en https://www.semana.com/opinion/articulo/carta-a-la-jepsobre-unas-mentiras/202000/.

26 Al respecto la reforma Tributaria puesta en marcha en 2019 buscó reducir la tributación a las empresas, bajo el argumento de que el país tenía una de las tarifas más altas para corporaciones, impulsando supuestamente el objetivo de estimular nuevas inversiones y evitar la salida de capital hacia jurisdicciones menos gravosas.

27 Aunque es anecdótico, fue ilustrativo el desconocimiento del coste de alimentos de la canasta familiar que denotó el exministro de Hacienda, Alberto Carrasquilla. Disponible en Forbes "Fuertes críticas a Carrasquilla por decir que una docena de huevos cuesta \$1.800", 19 de abril de 2021. Disponible en https://forbes.co/2021/04/19/actualidad/fuertes-criticasa-carrasquilla-por-decir-que-una-docena-de-huevos-cuesta-1$800 \%$.

28 Información publicada por Temblores ONG el 18 de mayo de 2021, a través de sus redes sociales. Disponible en https://www.temblores.org/.

29 Defensoría del Pueblo, “Defensor del Pueblo solicitó audiencia y entregó a CIDH balance sobre la situación de derechos humanos en el país", 25 de mayo de 2021. Disponible

https://www.defensoria.gov.co/es/nube/comunicados/101 49/Defensor-del-Pueblo-solicit\%C3\%B3-audiencia-yentreg\%C3\%B3-a-CIDH-balance-sobre-la-situaci\%C3\%B3n-dederechos-humanos-en-el-pa\%C3\%ADs-paro-nacionalDefensor\%C3\%ADa-CIDH.htm.

30 W Radio, "Es absolutamente desproporcionado el uso de la fuerza en las protestas: CIDH", 27 de mayo de 2021. Disponible

https://www.wradio.com.co/noticias/internacional/es-

absolutamente-desproporcionado-el-uso-de-la-fuerza-en-lasprotestas-cidh/20210527/nota/4138242.aspx. 